\title{
A CAVITATION TRACKING METHOD FOR ELASTIC-PLASTIC FLOW IN SOLIDS
}

\author{
Keh-Ming Shyue
}

Department of mathematics, National Taiwan University, Taipei 106, Taiwan (shyue@ntu.edu.tw)

\begin{abstract}
We present a front tracking approach for the numerical simulation of one-dimensional elasticplastic flow in solids with cavitation. The algorithm uses a simplified model system where the behavior of the materials is modeled by a barotropic equation of state for the hydrostatic pressure, the Hooke's law for the deviatoric stress, and the von Mises condition for the elastic-plastic phase change. To characterize the cavitation in the solution state, a simple cut-off criterion is employed. Sample numerical results are shown to validate the proposed front-tracking method.
\end{abstract}

Keywords:Front tracking method; Hooke's law; Von Mises yield condition; Cavitation

PACC:83.50.-v; 46.25.-y, 46.35.+z; 62.30.+d

\section{INTRODUCTION}

Our goal in this paper is to describe a front tracking approach for the efficient numerical simulation of dynamics' problems with cavitation that arise from elastic-plastic flow in solids. To demonstrate the basic idea, we use the mathematical model proposed by Wilkins [11] as an example in that the barotropic version of the governing equations in one dimension takes the form

$$
\left\{\begin{array}{l}
\frac{\partial \rho}{\partial t}+\frac{\partial}{\partial x}(\rho u)=0 \\
\frac{\partial}{\partial t}(\rho u)+\frac{\partial}{\partial x}\left(\rho u^{2}+\sigma_{x}\right)=0 \\
\frac{\partial s_{x}}{\partial t}+u \frac{\partial s_{x}}{\partial x}-\frac{4}{3} \mu \frac{\partial u}{\partial x}=0
\end{array}\right.
$$

Here $\rho$ is the density, $u$ is the particle velocity, $\sigma_{x}=p(\rho)-s_{x}$ is the total stress, $s_{x}$ is the deviatoric stress, and $\mu$ is the shear modulus. For simplicity, the behavior of solids at a high hydrostatic pressure, denoted by $p$, is assumed to be a function of the density only, see [3] for the more general constitutive laws. It should be mentioned that the first two equations in (1) are the conservation laws for the mass and momentum, and the last equation comes from the Hookes' law for the constitutive relation between the stress and strain rate.

To complete the model, the von Mises yield condition is employed to describe the elastic limit, and in one dimension this is that

$$
s_{x}^{2} \leq \frac{2}{3} Y_{0}^{2},
$$

where $Y_{0}$ is the yield strength of the material. Note that when inequality (2) is violated, plastic flow begins to occur, and for a perfectly-plastic flow (this is the case concerned here) the stress deviator is assumed to remain constant beyond this point (cf. [11] for the more details). Furthermore, if the total stress $\sigma_{x}$ drops to a critical value $\sigma_{\mathrm{V}} ; \sigma_{x} \leq \sigma_{\mathrm{V}}$, the flow state is said to be in a cavitation zone. In this work, we have chosen the data well so that there is no fracture zone present in the solution.

To handle the occurrence of cavitation boundaries in this elastic-plastic model, which is fundamental importance to many practical impact problems, we use a front tracking method developed previously by LeVeque and the author [6]. In the method, our grid consists of two parts. We choose a uniform, underlying grid with mesh size that remains fixed for all time, and we also introduce tracked points 
(the locations of cavitation boundaries in the current case) which vary from step to step. These tracked points subdivide some regular cells into two or more subcells, creating some irregular cells. High-resolution Godunov methods based on the large time step wave propagation approach are employed on the resulting nonuniform grid to update all the cell averages. Several calculations are presented below to demonstrate the effectiveness of the method.

\section{FRONT TRACKING METHOD}

In each time step, our front tracking method consists of the following steps:

1) Flag tracked cavitation boundaries by checking solutions of the cavitation-included Riemann problem; the location of the tracked interfaces at the next time step can be determined,

2) Modify the current grid by inserting these new tracked interfaces; some cells will be subdivided and the values in each subcell must be initialized,

3) Take a time step on this nonuniform grid using a high-resolution Godunov method to update the cell averages,

4) Delete the old tracked interfaces from the previous time step; some subcells will be combined and a value in the combined cell must be determined from the subcell values.

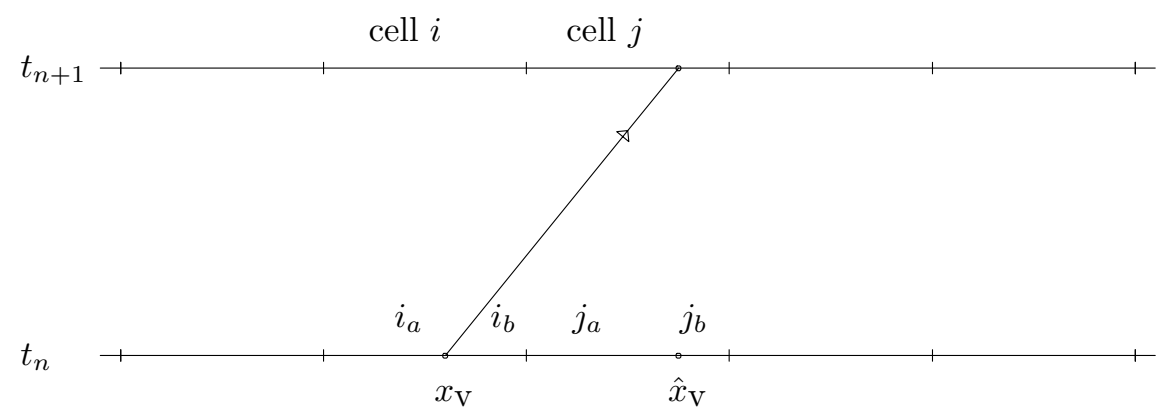

Figure 1: A tracked cavitation boundary at $x_{\mathrm{V}}$ propagating from cell $i$ to a new location at $\hat{x}_{\mathrm{V}}$ in cell $j=i+1$ leads to a subdivision of cells $i$ and $j$. In time step $n$ we split cell $j$ in two, and in time step $n+1$ we eliminate the old tracked point in cell $i$.

Figure 1 shows a typical example where a tracked cavitation boundary at $x_{\mathrm{V}}$ is advanced based on the solution of the cavitation-included Riemann problem (cf. $[8,9]$ ) from the current time to the next to $\hat{x}_{\mathrm{V}}$. In this instance, this new tracked boundary subdivides cell $j$ into two subcells $j_{a}$ and $j_{b}$, and we must assign a cell value to each of these subcells. This can be done by simply assigning the previous cell value to each subcell.

Once the new grid is constructed, the solution can then be updated using a a state-of-the-art numerical method for hyperbolic conservation laws. This method should be able to deal with the irregular cells near the tracked boundary. In particular, it must maintain stability, even if some of these cells are very small relative to the underlying mesh size used to determine the time step, and also hopefully to maintain second order accuracy in the smooth flow on either side. To accomplish this, we use a high resolution method based on the large time step wave propagation approach, developed by LeVeque [4]. The main idea is that waves arising from the solution of Riemann problems at the cell boundaries are propagated the appropriate distance determined by the wave speed and time step, and used to update cell averages in any grid cell they encounter. The wave may affect more than one cell if the neighboring cell is very small. In 
this manner, the stencil of the method adjusts automatically so that the CFL (Courant-Friedrich-Lewy) condition is always satisfied regardless of the configuration of the grid.

To end this time step, we need to delete the old tracked boundaries from the current grid such as $x_{\mathrm{V}}$ as shown in Fig. 1. This corresponds to merge two subcells into one, i.e., $i_{a}$ and $i_{b}$, and the cell value in the combined cell is calculated by the appropriate weighted combination of these two deleted subcells to maintain the correct cell average.

\section{NUMERICAL EXAMPLES}

To validate our numerical method, we begin by considering a Riemann problem of Tang and Sotiropoulos [9] that involves the dynamic formation of cavity inside a steel plate in addition to the elastic-plastic transition of the material. Initially, on the left the interface, $-5 \mathrm{~m} \leq x<0 \mathrm{~m}$, we use

$$
(\rho, u)_{L}=\left(8046.83 \mathrm{~kg} / \mathrm{m}^{3},-10^{-3} \mathrm{~m} / \mathrm{s}\right),
$$

and on the right of the interface, $0 \mathrm{~m} \leq x \leq 5 \mathrm{~m}$, we have

$$
(\rho, u)_{R}=\left(7755.74 \mathrm{~kg} / \mathrm{m}^{3}, 10^{-3} \mathrm{~m} / \mathrm{s}\right) .
$$

As in [9], the hydrostatic pressure of the steel is modeled by the Murnaghan (also called Tait) equation of state of the form

$$
p(\rho)=\mathcal{A}\left(\rho / \rho_{0}\right)^{\gamma}-\mathcal{B}
$$

with the parameter values: $\gamma=3.7, \rho_{0}=7.8 \mathrm{Mg} / \mathrm{m}^{3}, \mathcal{A}=60.1351 \mathrm{GPa}, \mathcal{B}=60.135 \mathrm{GPa}$, and the constitutive material quantities: $\mu=85.3 \mathrm{GPa}, Y_{0}=0.979 \mathrm{GPa}$, and $\sigma_{\mathrm{V}}=-20 \mathrm{GPa}$, for the current modelling of elastic-plastic flows with cavitation.

For this problem, it is known that the resulting solution consists of two leftward-going rarefaction waves (the faster rarefaction is an elastic precursor, while the slower one is a plastic wave), a rightwardgoing elastic shock wave, and a vacuum region lying in between that separates the rarefactions and the shock. We run this problem using the high-resolution method with the MINMOD limiter (cf. [5]), the Courant number 0.9 , and the mesh size $\Delta x=1 / 20 \mathrm{~m}$. Snapshots of the computed density, velocity, total stress, and the stress deviator are shown in Fig. 2 at time $t=507 \mu$ s where we have also included the fine grid solution obtained using the same method but $\Delta x=1 / 100 \mathrm{~m}$. From the figure, we clearly observe reasonable resolution of the solution structure without any spurious oscillations near the vacuum boundaries. When we make the direct comparison of our solutions with those appeared in [9], we find very good agreement of the results.

For many practical applications, it is of importance to see how the flow behavior is different if the material behaves entirely hydrodynamically, but not in an elastic-plastic manner. Here we perform such a computation using the same method as before, and present the result in Fig. 3. From there, it is easy to notice that the hydrodynamic shock is traveling slower than the elastic shock, and the step behind the faster rarefaction wave (see Fig. 2) is no longer existing in this hydrodynamic calculation. Note that this result shows that even though the yield strength $Y_{0}$ is smaller than the total stress $\sigma_{x}$, the influence on the wave can be very pronounced (see the results presented below also). It should be mentioned that in the above computations, there is no vacuum boundary in the domain initially, it has been introduced and tracked subsequently by the front-tracking method at any space and time when the cavitation condition $\sigma_{x} \leq \sigma_{\mathrm{V}}$ is satisfied. In the meantime, we have left the other waves captured.

We are next concerned with a popular flying aluminum-plate problem of Wilkins (cf. [10]) that incorporates a free surface and plasticity. To model the hydrostatic pressure of the aluminum, we use the polynomial equation of state of the form

$$
p(\rho)=\mathcal{A}\left(\rho / \rho_{0}-1\right)+\mathcal{B}\left(\rho / \rho_{0}-1\right)^{2}+\mathcal{C}\left(\rho / \rho_{0}-1\right)^{3}
$$



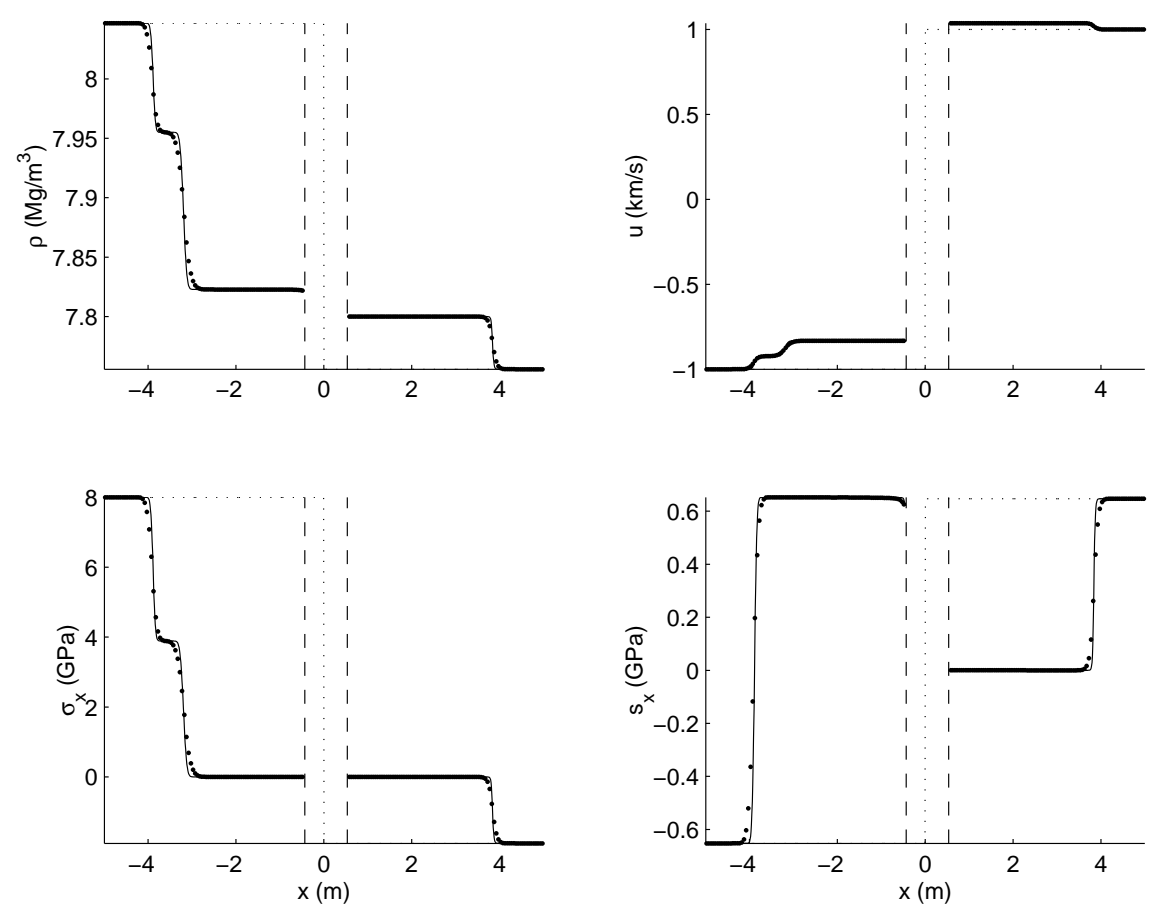

Figure 2: Elastic-plastic calculations for a Riemann problem with cavity inside a steel plate at time $t=507 \mu \mathrm{s}$. The solid line is the fine grid solution computed by $\Delta x=1 / 100 \mathrm{~m}$, and the points show the solution with $\Delta x=1 / 20 \mathrm{~m}$. The dashed line in eash subplot is the approximate location of the vacuum boundary, and the dotted line is the initial condition at time $t=0$.
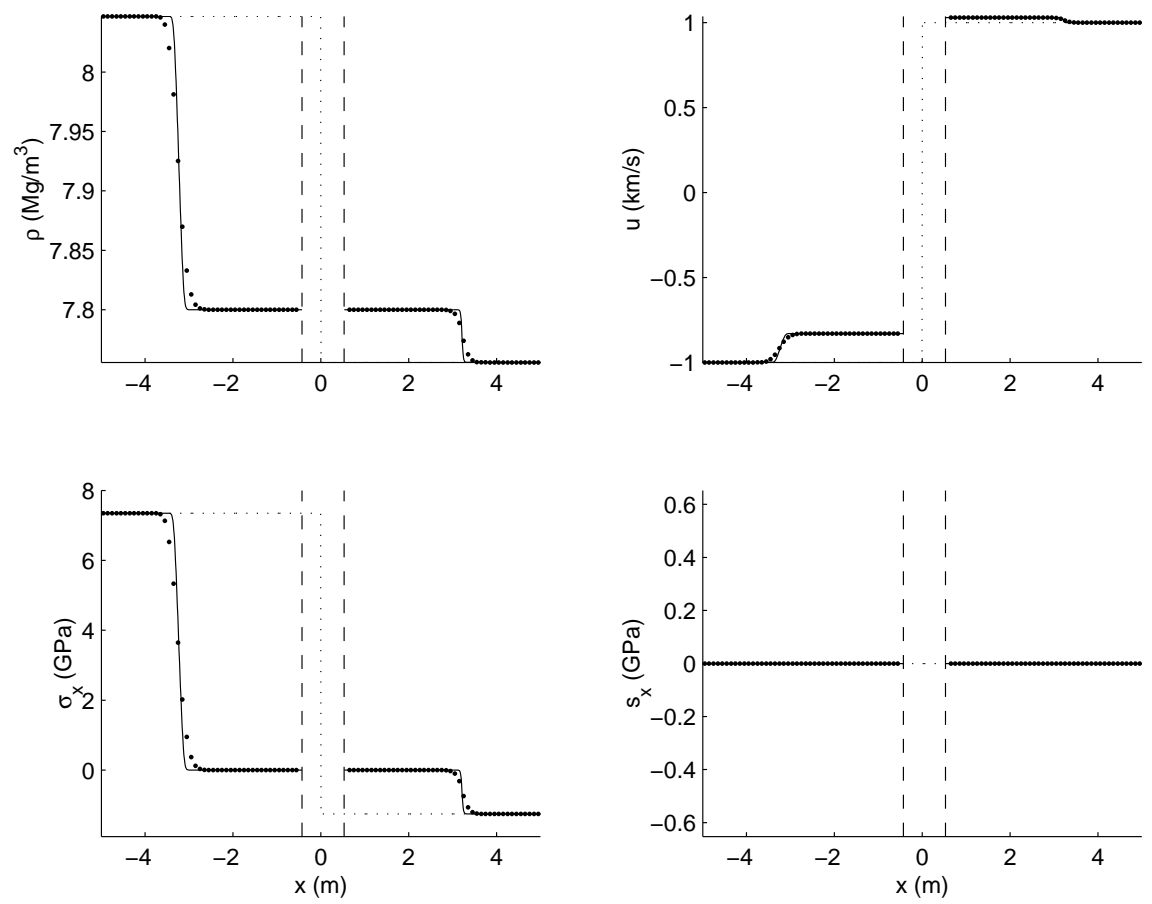

Figure 3: Hydrodynamic calculations for the same problem as considered in Fig. 2, but are run without the elastic-plastic yield condition. 
with $\rho_{0}=2.7 \mathrm{Mg} / \mathrm{m}^{3}, \mathcal{A}=73 \mathrm{GPa}, \mathcal{B}=172 \mathrm{GPa}$, and $\mathcal{C}=40 \mathrm{GPa}$, and take $\mu=24.8 \mathrm{GPa}$ and $Y_{0}=$ 0.2976 GPa in our model for elastic-plastic flow in solids. As for the initial condition, we have

$$
(\rho, u)=\left\{\begin{array}{cl}
\text { vacuum } & \text { for } x<1 \mathrm{~mm} \\
\left(\rho_{0}, u_{0}\right) & \text { for } 1 \mathrm{~mm} \leq x<5 \mathrm{~mm} \\
\left(\rho_{0}, 0\right) & \text { for } 5 \mathrm{~mm} \leq x \leq 50 \mathrm{~mm}
\end{array}\right.
$$

with $u_{0}$ denoted the initial impact velocity of the moving aluminum plate.

We run this problem using exactly the same high-resolution method as performed in the pervious example, but using a different $\Delta x=1 / 10 \mathrm{~mm}$. Results for the elastic-plastic flow calculations with $u_{0}=0.8 \mathrm{~km} / \mathrm{s}$ and $2 \mathrm{~km} / \mathrm{s}$ are shown in Figs. 4 and 5 , respectively. In the case of $0.8 \mathrm{~km} / \mathrm{s}$ (see Fig. 4), we observe a plastic shock wave trailing behind an elastic shock precursor, while in the case of $2 \mathrm{~km} / \mathrm{s}$ (see Fig. 5), since the shock stress-deviator is above the elastic limit, we only see the plastic shock. In both cases, when the leftward-going shock waves reach the free surface, a leading rightward-going elastic rarefaction is formed, followed by the plastic rarefaction wave. As the time goes on, these waves begin to overtake the initial rightward-going shocks. We find good qualitative agreement of our results with the ones shown in $[7,10]$.

\section{CONCLUSIONS}

We have described a wave propagation method for the numerical simulation of a model elastic-plastic flow in solids in one dimension. Numerical results presented in this paper give a preliminary validation of the method. In the future, we plan to extend the method to more than one dimension, and also include more general constitutive laws for the simulation of practical impact problems (cf. $[1,2,7,11])$.

\section{ACKNOWLEDGMENTS}

The contents presented in this paper was (partially) supported by the Research Grants from National Science Council of Taiwan NSC 94-2115-M-002-016.

\section{REFERENCES}

1. N. Favrie, S. L. Gavrilyuk, and R. Saurel, J. Comput. Phys., 228, 6037-6077 (2009).

2. B. P. Howell and G. J. Ball, J. Comput. Phys., 175, 128-167 (2002).

3. A. G. Kulikovskii, N. V. Pogorelov, and A. Yu. Semenov, Mathematical aspects of Numerical Solution of Hyperbolic Systems, Boca Raton: Chapman \& Hall/CRC, 2001.

4. R. J. LeVeque, J. Comput. Phys., 78, 36-63 (1988).

5. R. J. LeVeque, Finite Volume Methods for Hyperbolic Problems, Cambridge: Cambridge University Press, 2002.

6. R. J. LeVeque and K.-M. Shyue, SIAM J. Sci. Comput., 16, 348-377 (1995.

7. G. H. Miller and P. Colella, J. Comput. Phys., 167,131-176 (2001).

8. H. S. Tang and D. Huang, J. Comput. Phys., 128, 301-318 (1996).

9. H. S. Tang and F. Sotiropoulos, J. Comput. Phys., 151, 790-815 (1999).

10. M. L. Wilkins, "Calculation of elastic-plastic flow", Methods in Computational Physics, Vol. 3, edited by B. Adler, S. Fembach, and M. Rotenberg, New York: Academic Press, 1964, pp. 211-263.

11. M. L. Wilkins, Computer Simulation of Dynamic Phenomena, New York: Springer, 1999. 

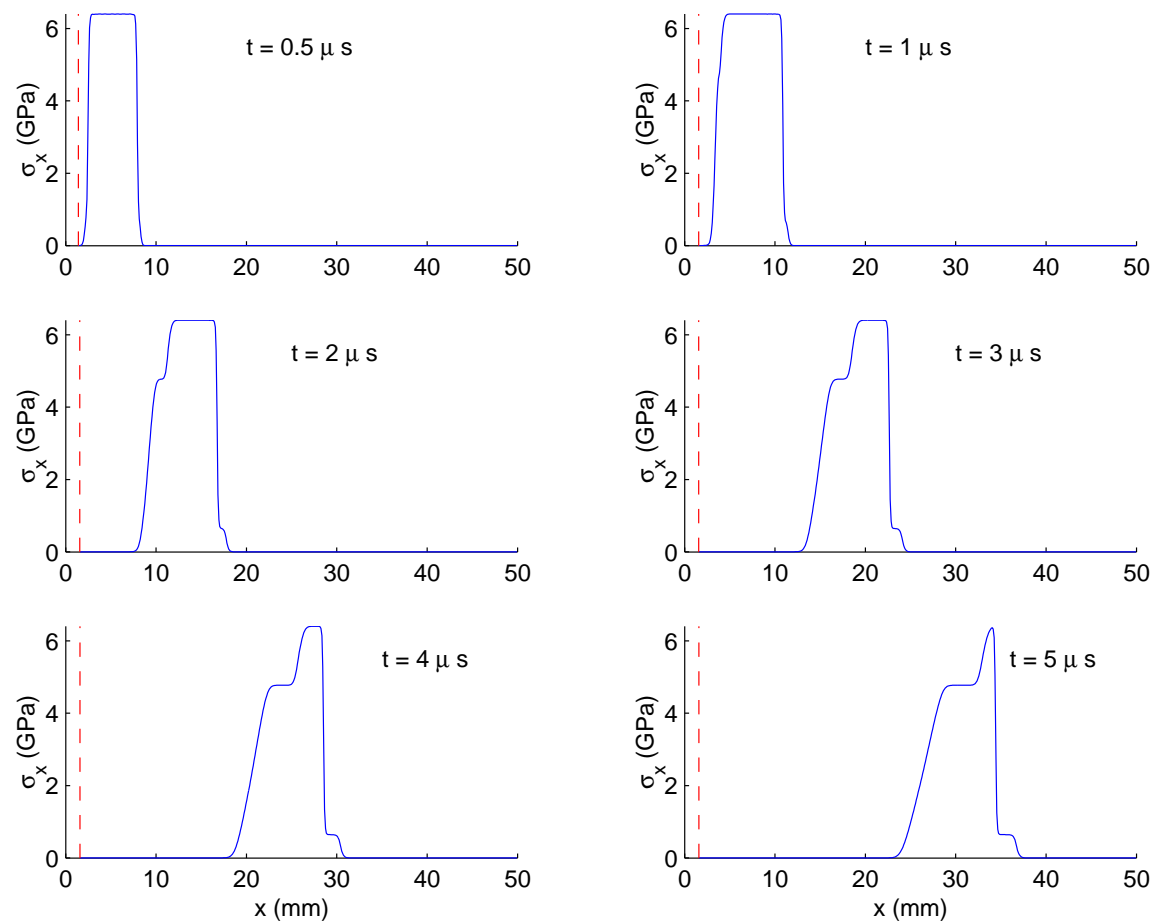

FiguRE 4: Elastic-plastic flow calculations for Wilkins' problem with impact velocity $0.8 \mathrm{~km} / \mathrm{s}$. Total stress $\sigma_{x}$ is shown at six different times $t=0.5,1,2,3,4$, and $5 \mu$ s. The dashed line in each subplot is the approximate location of the free surface.


FiguRE 5: Elastic-plastic flow calculations for Wilkins' problem with impact velocity $2 \mathrm{~km} / \mathrm{s}$. The graphs of the solutions are displayed in the same manner as in Fig. 4. 